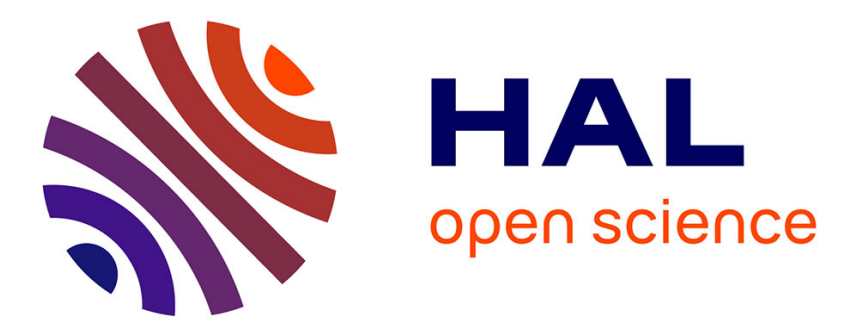

\title{
Dislocation dynamics during cyclic loading in copper single crystal
}

Gabriel L'Hôte, Sophie Cazottes, J. Lachambre, M. Montagnat, P. Courtois, Jérôme Weiss, Stéphanie Deschanel

\section{To cite this version:}

Gabriel L'Hôte, Sophie Cazottes, J. Lachambre, M. Montagnat, P. Courtois, et al.. Dislocation dynamics during cyclic loading in copper single crystal. Materialia, 2019, 8, pp.100501. 10.1016/j.mtla.2019.100501 . hal-03001659v2

\section{HAL Id: hal-03001659 \\ https://hal.science/hal-03001659v2}

Submitted on 9 Mar 2021

HAL is a multi-disciplinary open access archive for the deposit and dissemination of scientific research documents, whether they are published or not. The documents may come from teaching and research institutions in France or abroad, or from public or private research centers.
L'archive ouverte pluridisciplinaire HAL, est destinée au dépôt et à la diffusion de documents scientifiques de niveau recherche, publiés ou non, émanant des établissements d'enseignement et de recherche français ou étrangers, des laboratoires publics ou privés.

\section{(c)(1)}

Distributed under a Creative Commons Attribution| 4.0 International License 


\title{
Dislocation dynamics during cyclic loading in copper single crystal
}

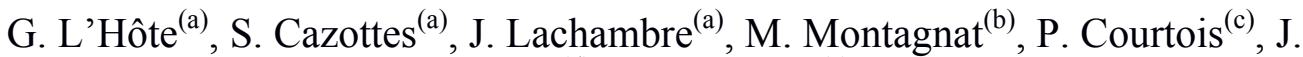 \\ Weiss $^{(\mathrm{d})}$, S.Deschanel ${ }^{(\mathrm{a})}$
}

(a) Université de Lyon, INSA-Lyon, Université Claude Bernard Lyon 1, CNRS, Laboratoire MATEIS, 7 avenue Jean Capelle, 69621 Villeurbanne Cedex, France

(b) IGE, Université Grenoble Alpes, CNRS, IRD, G-INP, 38041 Grenoble, France

(c) Institut Laue Langevin, 6, rue Jules Horowitz - 38042 Grenoble, France

(d) ISTerre, Université Grenoble Alpes, F-38400 Grenoble, France

gabriel.1-hote@insa-lyon.fr

\begin{abstract}
Crystalline plasticity can take place through numerous, small, uncorrelated dislocation motions (mild plasticity) or through collaborative events: dislocation avalanches (wild plasticity). Here, we study the correlation between dislocation patterning under cyclic loading and the nature of collective dislocation dynamics. The dislocation motion of a [110] oriented pure copper single crystal was dynamically followed using Acoustic Emission (AE) for different imposed stress amplitudes. The dislocation structure between each cyclic stress step was investigated using Electron BackScattered Diffraction (EBSD) and Rotational-Electron Channeling Contrast Imaging (R-ECCI) in a Scanning Electron Microscope (SEM). At low imposed stress, when the structure consists of dislocation cells, few dislocation avalanches are observed, while for a wall structure, at higher imposed stress, the contribution of avalanches is increased during the first cycles. For a given stress amplitude, the evolution of mild plasticity is synchronous with the plastic strain-rate, and rapidly vanishes after few cycles due to work hardening. The mean free path of the dislocations in this mild plasticity regime corresponds to the characteristic size of the dislocation structure (cell size, distance between walls). From one stress level to another, brutal rearrangements of the dislocation structure occur within a few numbers of cycles. Those rearrangements take place, at least partly, through dislocation avalanches. Upon reloading at a larger stress amplitude, dislocation avalanches can travel over distances much larger than the former dislocation mean free path. As the dislocation avalanches spread within the crystal, the memory of the previous dislocation structure is lost and a new dislocation structure emerges.
\end{abstract}

Keywords: Acoustic methods; Dislocation Dynamics; Copper; Fatigue; Electron Channeling Contrast Imaging

This version corresponds to the final version as published in Materialia (https://doi.org/10.1016/j.mtla.2019.100501). 


\section{Introduction}

Depending on the material and the loading history, plasticity dynamics can be homogeneous in both time and space, taking place in the form of numerous, small, and uncorrelated dislocation motions (mild plasticity). In pure metals, these dislocation motions are limited by the surrounding dislocation structure [1,2], characterized by internal characteristic lengths and emerging itself from short-range interactions between dislocations [3-5]. The dislocation mean free path $\lambda$ represents the distance over which a dislocation can travel before being stopped by dense dislocation arrangement (cells, walls, high angle sub-boundaries, etc.). $\lambda$ can be determined directly from Transmission Electron Microscope (TEM) or Electron Channeling Contrast Imaging (ECCI) in the Scanning Electron Microscope (SEM) $[2,6]$. Alternatively, plasticity can be highly intermittent in the form of scale-free dislocation avalanches that are inhomogeneous both in time and space (wild plasticity) [7,8]; these avalanches consist of highly correlated dislocation motions that collectively move in the crystal [9-11]. It has recently been demonstrated that mild and wild plasticity can coexist during plastic deformation [7]. The wildness $(W)$ is then defined as the proportion of plastic deformation accounted by dislocation avalanches [12]. In pure materials, the relative occurrence of these two types of plasticity depends on the presence of a dislocation structure, a dense dislocation structure being assumed to restrict the extension and the role of dislocation avalanches [7]. The formation of the dislocation structure, in turn, is related to the crystallographic nature of the material and the loading mode. In particular, for crystal structures favoring short-range interactions between dislocations (e.g. the facecentered cubic (FCC) structure), a dislocation structure emerges during plastic deformation. The wild fluctuations are then limited and plastic deformation is mainly accommodated through mild fluctuations. In contrast, in crystal structures favoring long-range interactions between dislocations (such as ice or HCP crystals), where no stable dislocation structure formation is observed, intermittency is preeminent $[7,13]$. Indeed, past acoustic emission (AE) experiments performed on both single and polycrystals of HCP metallic materials and ice during monotonic loading revealed that the plastic deformation processes are not homogeneous in time and space. Instead, they are governed by avalanches of dislocations moving through the material in a very heterogeneous and intermittent fashion, with nearly $100 \%$ of the deformation accommodated by dislocation avalanches $[8,11,14]$. The same findings were observed in discrete and continuous numerical simulations $[9,10,15]$. Yet, these results were mainly obtained under monotonic loading. Hence we question the influence of cyclic loading on the coexistence between mild and wild plasticity

Cyclic loading of monocrystalline materials has been studied for more than 50 years. The evolution of mechanical behavior and the emergence of the dislocation structure under cyclic fatigue loading has mainly been studied under strain-controlled mode [16-23]. The material generally presents a hardening stage, followed by softening (observed in some cases) and a saturation stage, which may be followed by a secondary hardening [24]. These stages (observed by the evolution of $\sigma_{\max }$ at each cycle) are the macroscopic signature of the internal evolution of the dislocation structure. The hardening stage is characterized by a rapid multiplication of 
dislocations and the formation of the first dislocation entanglements. When the dislocation structure formed during the hardening stage is not able to accommodate the imposed strain, the crystal proceeds to local rearrangements of its structure. These rearrangements then facilitate plastic flow, leading to a reduction of $\sigma_{\max }$ : the softening stage. Finally, the saturation stage corresponds to a balance between multiplication and annihilation of dislocations composing the structure. The cyclic saturation is only macroscopic and the microstructure can always evolve through cycles. These different stages can also be observed during stress-imposed cyclic loading [25-30]. While the test control mode (stress-imposed vs strain-imposed) has an influence on the mechanical response of the material, similar dislocation arrangements can be obtained [31,29,32].

In both fatigue control modes, the evolution of the mechanical response seems to be smooth, i.e, without stress drops or strain burst, as illustrated by the very gradual evolution of the stress during strain-imposed cyclic loading, or of the strain is stress-imposed cyclic loading. To the authors' knowledge, only few references are available about the relation between the nature of the dislocation structure and the occurrence of dislocation avalanches during cyclic loading [33-37].

Neumann $[33,34]$ observed for the first time the presence of strain bursts during a stress ramp cyclic test on copper single crystals. These strain bursts convey a significant increase in plastic deformation over a period of about 50 consecutive cycles and are assumed to come from a chain collapse, in the form of dislocation avalanches, of the entire dislocation structure. Strain bursts are then a macroscopic representation of many collaborative motions. However, this work does not allow a more detailed study of the avalanches that compose these strain bursts. In the early 1980's, Yan and Laird [36] conducted cyclic loading tests on pure copper (FCC) single crystals using high-frequency recording of the hysteresis loops, while the dislocation structure was characterized using Transmission Electron Microscopy. They concluded that dislocation avalanches were associated with the presence of persistent slip bands (PSBs), from the low-resistance glide of screw dislocations within these bands. In our study, however, no PSBs have been observed. In a recent work [12], collective dislocation dynamics during the strain-controlled cyclic loading of pure $\mathrm{Al}$ (FCC crystal) was investigated by means of acoustic emission (AE). It was shown in particular, that the wild component of plasticity rapidly faded away with cycling, presumably as the result of the emergence of an increasingly stable dislocation structure frustrating dislocation avalanches. However, these AE measurements were not associated with microstructural analyses.

In the present work, we investigated the plasticity dynamics (mild vs. wild fluctuations) in pure copper single crystal under stress-imposed cyclic loading, by means of $\mathrm{AE}$, in combination with the characterization of dislocation structures from ECCI.

If TEM and ECCI provide good insights about dislocation structures at a given time (the type of dislocation structure and quantification of $\lambda$ ), those methods are not adapted for the dynamical study of dislocation motion, especially during cyclic loading. On the other hand, the AE technique is particularly powerful for 
studying real-time crystalline plasticity phenomena [38-40]. This technique consists of recording the transient waves produced by the sudden release of elastic energy. The electrical signals generated during irreversible processes can be divided into two categories: the so-called continuous-AE, which is the signature of mild plasticity, and the discrete-AE type, which is represented by acoustic bursts and related to wild plasticity [7]. Therefore, AE can be used to distinguish between these two types of plasticity during mechanical tests. Thus, the combination of AE and ECCI will allow quantifying the relationship between dislocation structures and dislocation dynamics.

We chose to study pure copper single crystal because it is a well-documented model material. In addition, the absence of precipitates or phase transformation restricts $\mathrm{AE}$ sources to dislocation motion and mutual interactions of the dislocations or microcracking. We ascertained the absence of microcracking for the test presented in this study. We studied the evolution of mild and wild plasticity during cycling at a given cyclic stress amplitude and at different increasing imposed stress amplitudes. In addition to the real-time study of the deformation processes, dislocation structures were characterized before and after each cyclic stress step using the Rotational-ECCI (R-ECCI) method [41]. Complementary electrons backscattered diffraction (EBSD) characterizations were performed to monitor the global disorientation and its evolution. This way, we can relate the nature of plasticity, quantified by its wildness, to the nature of the dislocation structure and its characteristics (dislocation density, dislocation mean free path, etc.).

\section{Material and Instrumentation}

Pure copper single crystal $(99.999 \%)$ was elaborated using the Bridgman method [42] (at Institut Laue Langevin (ILL), Grenoble), and the specimen was cut using electro-erosion. The specimen was oriented close to [110] in the tensile direction (TD) and along [111] and [112] in the two normal directions, favoring multiple slip on $\{111\}<110>$ slip systems (with Schmid factors M equal to 0.41 ). The sample was mechanically ground, with final polishing performed using a $1-\mu \mathrm{m}$ diamond solution. Because of the high ductility of the specimen, electropolishing was performed in D2 electrolyte (Struers APS, Denmark) at $20 \mathrm{~V}$ for $20 \mathrm{~s}$ at $5^{\circ} \mathrm{C}$ using a LectroPol-5 device (Struers APS, Denmark) to prevent strain hardening by the initial polishing.

Stress-controlled cyclic steps were performed on the same sample, using a hydraulic fatigue machine that was specifically designed to generate low noise (Figure 1(a)). Because of the high ductility of copper single crystal, classical contact extensometers could not be used, as their use would induce localized damage on the surface as well as acoustic noise that could be recorded by the AE set-up. To measure the specimen strain, we performed digital image correlation (DIC). This deformation measurement by DIC does not allow us to perform cyclic loading under strain control mode yet. Works are in progress to perform such strain-controlled cyclic tests. DIC enables displacement field measurements $u(x)$ to be performed at the specimen surface, assuming conservation of the optical flow, between an image of reference and a deformed image. In this study, DIC was used as a strain gauge: a region of 
interest (ROI) of $\sim 3.3 \times 3.8 \mathrm{~mm}$ was defined as the gauge, with the strain tensor being the expected result. Images were recorded using a camera (mvBlueFOX 124C, MATRIX VISION) at a frequency of $3.2 \mathrm{~Hz}$. Only the mean axial strain $E_{y y}$ (average of the values in the ROI) is presented in what follows. The strain resolution was approximately $0.002 \%$. To attain sufficient contrast, a speckle pattern was painted on the specimen surface using black and white paint (Figure 1(b)).

Several cyclic steps were performed by increasing the stress amplitude on the very same copper specimen. A loading ratio of $R_{\sigma}=\sigma_{\min } / \sigma_{\max }=0.1$ and a loading frequency of $0.1 \mathrm{~Hz}$ were fixed for each step. The imposed resolved shear stress (RSS) (on $\{111\}$ glide planes) amplitude was 1-10 MPa, 1.5-15 MPa, 2-20 MPa, 2.5-25 MPa, and 3-30 MPa for the five steps, as shown in Figure 1(c). A total of 5000 cycles were performed for each stress step, ensuring that the crystal was in a stable state at the end of each step. Because of experimental issues during the 2-20 MPa cyclic loading (decoupling of the acoustic sensors), the corresponding AE results are not presented. Consequently, only the microstructural characterization is presented for this stress step. The crosses in Figure 1(c) indicate when the R-ECCI and the EBSD characterization were performed.

ECCI is a technique performed in a scanning electron microscope, and its efficiency for dislocation characterization of bulk samples has been previously demonstrated $[43,44]$. This technique is increasingly used because of the simplified and non-destructive sample preparation compared with that for TEM analysis. For both TEM and ECCI, dislocations are visible when the crystal is slightly deviated from the "two-beam" condition orientation. This condition consists of fulfilling the Bragg condition for one family of planes by tilting and rotating the sample relative to the electron beam. As this orientation can be difficult to achieve, we recently developed a novel approach based on the rotation-acquisition of backscattered electron (BSE) images. In such an image series, specific angles should result in adequate two-beam conditions to observe dislocations. EBSD maps were obtained using an Oxford Instrument Symmetry detector. Dislocation observations were conducted on a classical SEM (Gemini I column, Carl Zeiss Microscopy GmbH, Germany)). The EBSD acquisition and R-ECCI observation conditions are described in L'Hôte et al. [41]. In this configuration, the spatial resolution achievable by ECCI is $10-20 \mathrm{~nm}[44]$.

a)

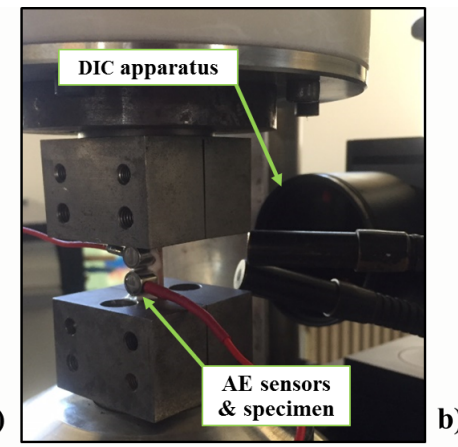

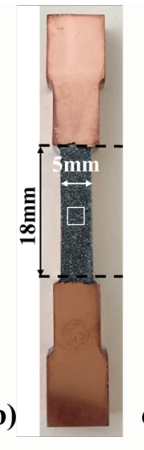

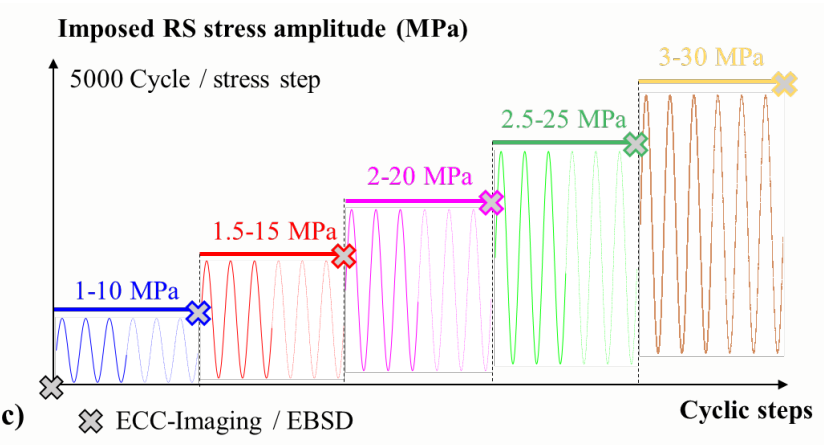


Figure 1. (a) Cyclic fatigue machine with DIC apparatus. (b) Photograph of the copper specimen used in this study with the typical speckle pattern on the gauge length. (c) Stressimposed fatigue steps.

The AE signal was continuously monitored using a Euro Physical Acoustics SA (EPA) PCI-2 Mistras data acquisition system with 40-dB pre-amplification and $50 \mathrm{kHz}-1.2 \mathrm{MHz}$ bandwidth. Two piezoelectric sensors (Nano30 EPA, 125-750 kHz bandwidth, resonance peak at $300 \mathrm{kHz}$ ) were glued on the sample surface using caf4® silicone glue. The raw AE signal was sampled at a frequency of $5 \mathrm{MHz}$. A representative raw AE signal is shown in Figure 2(a) for a 1s period of the first cycle of a cyclic test on a copper sample. Individual bursts, i.e. the so-called discrete AE signature of dislocation avalanches [7], were automatically identified by thresholding of this raw signal above $0.06 \mathrm{~V}(36 \mathrm{~dB})$, and their characteristics (e.g., arrival time, energy, duration) recorded. This standard protocol is detailed in [45]. In the meantime, the AE power $d E / d t$ was sampled continuously throughout the stress steps at a sampling frequency of $100 \mathrm{~Hz} . d E$ is given by the integral of the squared voltage signal over a period $d t=10 \mathrm{~ms}$. This $\mathrm{AE}$ power encompasses a discrete $\mathrm{AE}$ as well as a continuous AE including an instrumental/environmental noise and a noise component generated by the cumulative effect of multiple but uncorrelated small dislocation motions (10). Figure 2(b) shows the correspondence between the acoustic power and the raw signal (Figure 2(a)), where large bursts correspond to discrete AE.
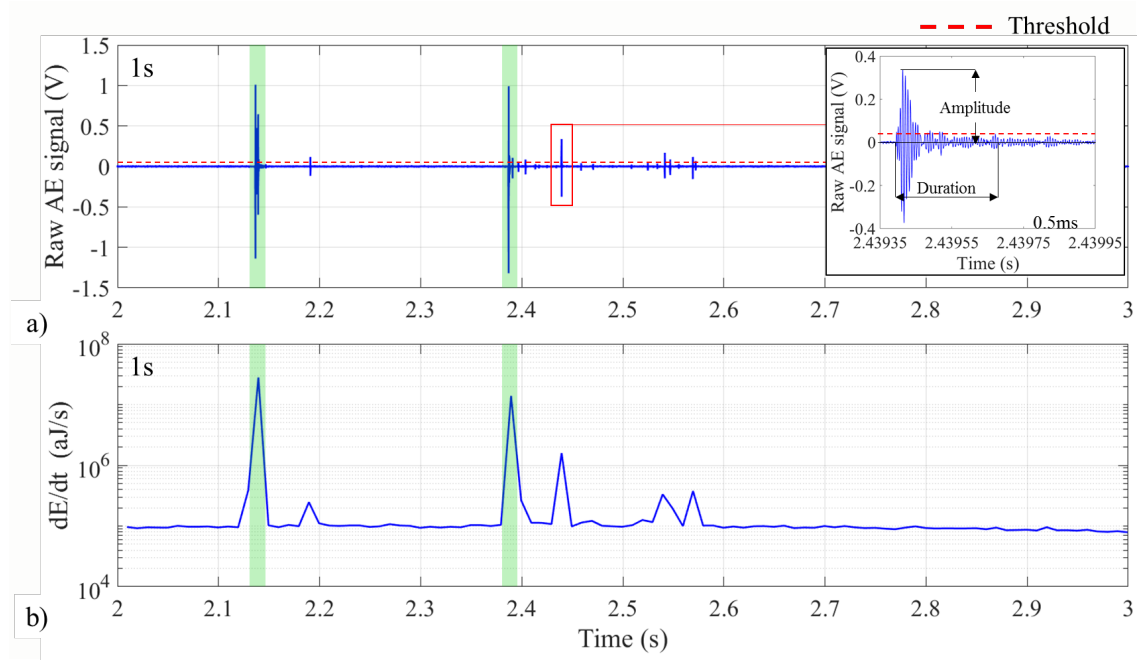

Figure 2. AE activity during $1 \mathrm{~s}$ of the first cycle of a stress-imposed fatigue test at 1-10MPa RSS. (a) Raw AE signal sampled at $5 \mathrm{MHz}$. An enlargement of a discrete AE waveform (detected above the voltage threshold (36 dB/0.06 V, red dashed line) is shown in the inset. (b) Evolution of AE power (aJ/s). Green areas show how bursts stand out in both the raw AE signal and the acoustic power.

In the studied copper single crystal, the only possible AE sources were limited to dislocation motion and microcracking. We ascertained the absence of microcracking from optical observations after each cyclic step, as well as the absence of AE multiplets, discrete acoustic emissions specific to fatigue crack growth, recently reported by Deschanel et al. [45]. Moreover, to obtain recordable AE signals originating from irreversible processes such as dislocation motion (mild plasticity and 
dislocation avalanches) and not just a steady background noise (electrical or external environment noise), the simultaneous motion of several dislocations is necessary. Mathis and Chmelik [46] estimated that a minimum of 10-100 dislocations must simultaneously move to produce sufficient energy for detection by AE sensors.

The fraction of plastic deformation accommodated by dislocation avalanches (wildness degree of plasticity) was evaluated upon cyclic loading while the dislocation substructure evolved. We define an AE proxy of wildness denoted by $W_{A E}$ as the fraction of $\mathrm{AE}$ energy released through detected $\mathrm{AE}$ bursts, after removal of instrumental/environmental noise [12]. $W_{A E}$ is a useful dimensionless measure of the plastic intermittency in bulk samples, especially when tracking its evolution over successive cycles.

\section{Results}

\subsection{Mechanical and AE response of copper single crystal deformed by cyclic loading}

In this section, the results for one stress-controlled cyclic step are presented in detail. For pedagogical reasons, the step at the RSS amplitude of 1.5-15 MPa (Figure $1(\mathrm{c})$, second step) is selected. A total of 5000 cycles lead to a final plastic strain of $\sim 2.6 \%$ at the end of the cyclic step. The plastic strain $\varepsilon_{\mathrm{pl}}$ was obtained by removing the elastic strain from the total strain, using Hooke's law $\varepsilon_{e l}=\sigma / Y$ with $Y$ the Young modulus $=137 \mathrm{GPa}[47,48]$. Figure 3 shows several stress-strain cycles for the cyclic stress step. The stress presented in the figure is the macroscopic imposed stress leading to a RSS of 1.5-15 MPa. The plastic deformation is very important during cycle $\mathrm{n}^{\circ} 1$ and is quickly reduced after cycle ${ }^{\circ} 5$ (pink curve).

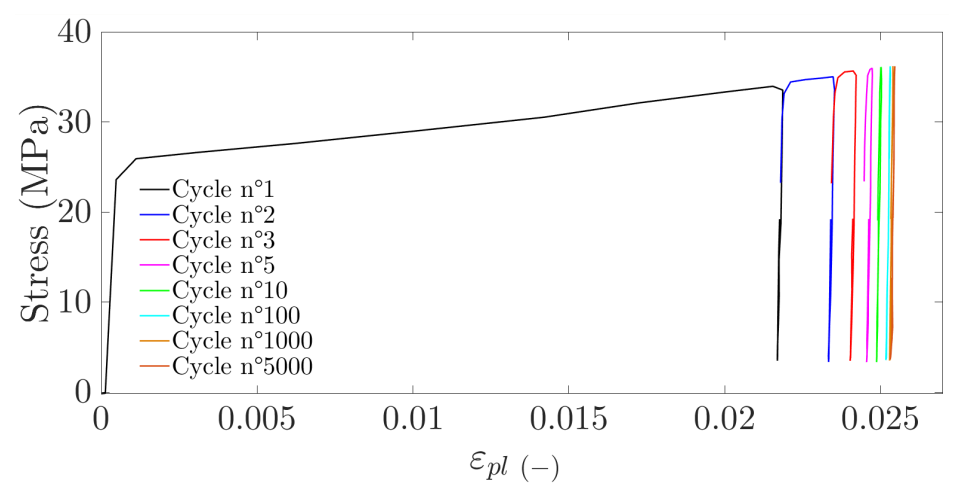

Figure 3. Several stress-strain cycles for the fatigue step at 1.5-15 MPa RSS. The stress displayed is the macroscopic stress $\left(\boldsymbol{\sigma}_{\boldsymbol{R} S \boldsymbol{S}}=\boldsymbol{M} * \boldsymbol{\sigma}_{\text {macro }}\right)$ with $M=0.41$ (Schmid factors) and the strain is the plastic strain $\left(\boldsymbol{\varepsilon}_{\boldsymbol{p}} \nu\right.$.

Figure 4(a) shows the cyclic stress amplitude and the associated plastic strain evolution for the first four cycles of the step. The plastic deformation of the sample rapidly decreased until becoming negligible, with most of the deformation occurring in the very first cycles. The red dots in Figure 4(a) indicate the stress and time at which AE bursts were detected; the AE bursts appeared mainly during the first two cycles. The acoustic power $d E / d t$ and plastic strain rate $\dot{\varepsilon}_{p l}$ are plotted in Figure 
4(b) (red and yellow curves, respectively). For clarity, only the signal from one of the two sensors is shown. The plastic strain rate shows a maximum during the first cycle. For the subsequent cycles, the maximum plastic strain rate greatly decreased until the plastic strain completely disappeared after approximately $4-5$ cycles.

The acoustic power (Figure 4(b)) consists of both an evolving continuous AE and acoustic bursts. The continuous part of the acoustic power (continuous part of the red curve) increased and decreased similarly to the evolution of the plastic strain rate. The AE power reached a maximum in the first cycle and then rapidly decreased during the subsequent cycles until the signal became flat. Similarly, the acoustic bursts were mainly generated during the first cycle ( $93 \%$ of the bursts) and especially when $\dot{\varepsilon}_{p l}$ was large. After the first cycle, the number and energy of the dislocation avalanches decreased, although some discrete $\mathrm{AE}$ bursts were recorded between cycle $\mathrm{n}^{\circ} 2$ and cycle $\mathrm{n}^{\circ} 83$ ( $\sim 7 \%$ of the total bursts).

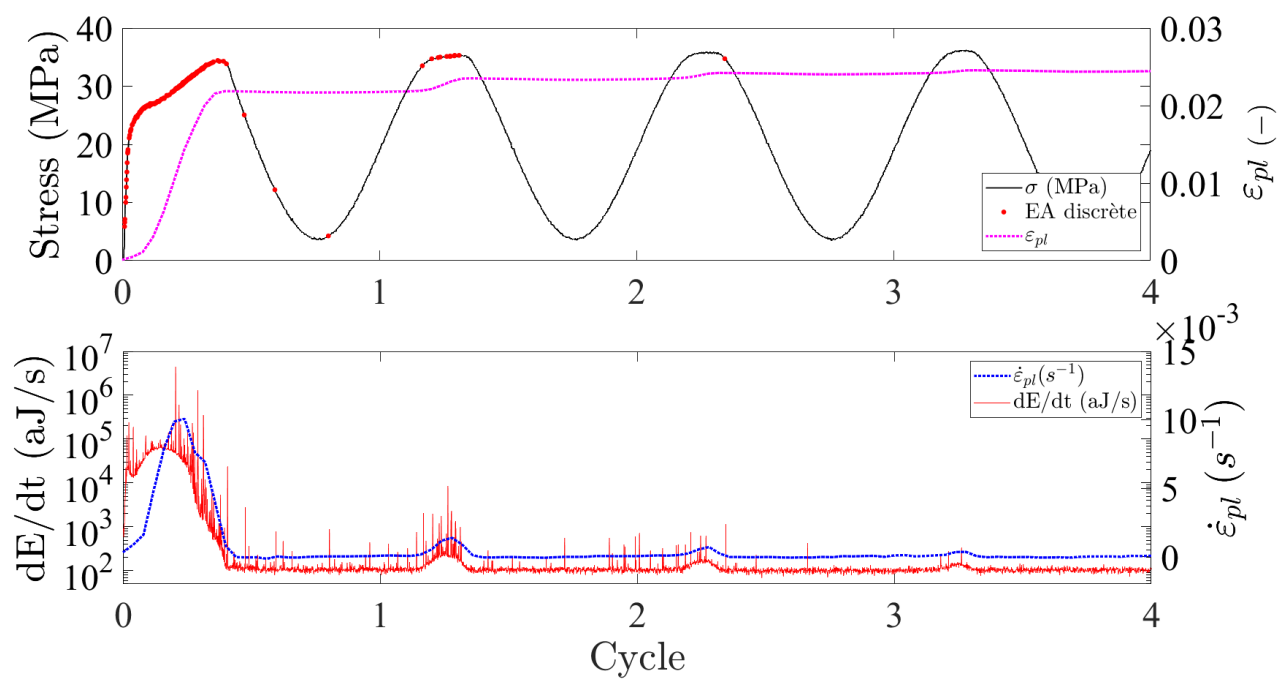

Figure 4. Evolution of AE over the first four cycles of the 1.5-15 MPa RSS cyclic step. (a) Stress (black) and $\boldsymbol{\varepsilon}_{\boldsymbol{p l}}$ (pink dashed line). The red dots indicate the level of stress at which $A E$ bursts were detected. (b) $\boldsymbol{d} \boldsymbol{E} / \boldsymbol{d} \boldsymbol{t}(a \mathrm{~J} / \mathrm{s})$ and associated $\boldsymbol{\varepsilon}_{\boldsymbol{p l}}^{\cdot}\left(s^{-1}\right)$.

Figure 5 shows the evolution of the wildness $W_{A E}$ (average value for each cycle) and the plastic strain amplitude $\Delta \varepsilon_{p l}\left(\varepsilon_{p l \text { max }}-\varepsilon_{p l \text { min }}\right)$ over 5000 cycles for the 1.5-15 MPa fatigue step. A strong correlation was observed between $W_{A E}$ and $\Delta \varepsilon_{p l}$. The wildness reached a maximum in the first cycle, with $\sim 20 \%$ of the energy originating from dislocation avalanches, and rapidly decreased to $0-1.5 \%$ after three cycles. 


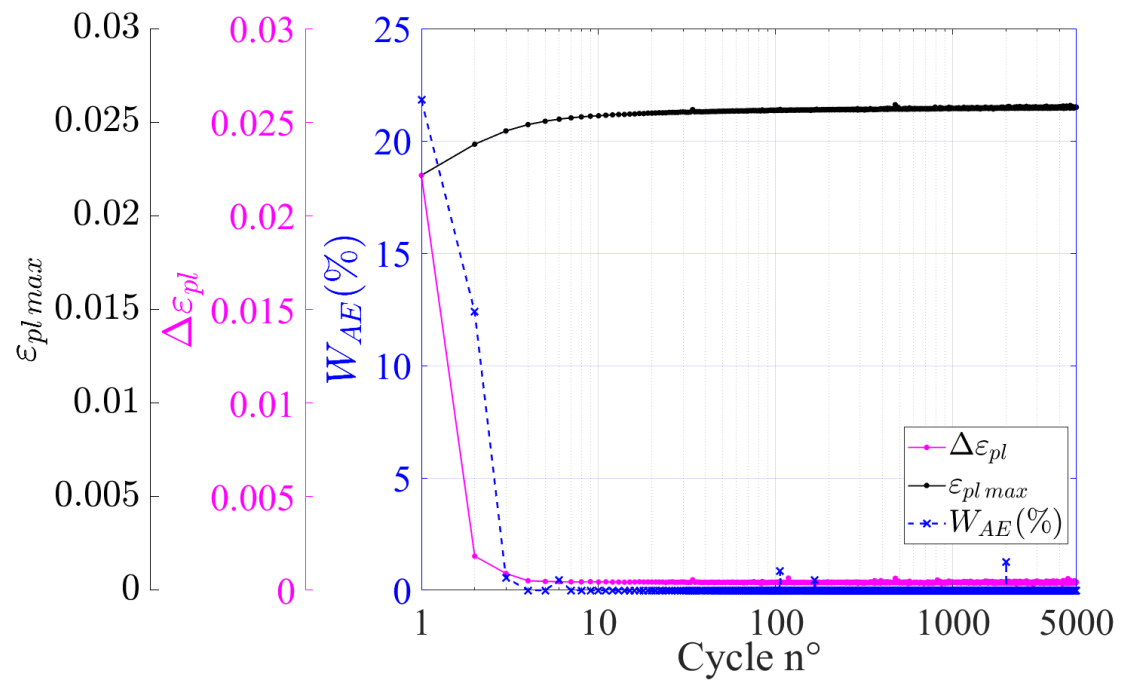

Figure 5. Evolution of the maximum of plastic strain, the plastic strain amplitude $\Delta \boldsymbol{\varepsilon}_{\boldsymbol{p l}}$ and the wildness $W_{A E}(\%)$, per cycle over 5000 cycles for the 1.5-15 MPa RSS cyclic step.

\subsection{Evolution of $\mathrm{AE}$ with the different stress steps}

\subsubsection{Correlation between strain and $\mathrm{AE}$}

Figure 6(a) presents the imposed stress and $\dot{\varepsilon}_{p l}$ for the first four cycles of the 1-10 MPa, 1.5-15 MPa, 2.5-25 MPa, and 3-30 MPa stress steps. Discrete AE signals within the cycle at different levels of stress (dots) are indicated on the stress curves. These signals were mainly generated during the first cycle, regardless of the imposed stress. Numerous bursts were recorded during the second cycle for the 2.5-25 and 3$30 \mathrm{MPa}$ steps, unlike for the 1-10 and 1.5-15 MPa steps. For the 3-30 MPa step, unlike for the other stress steps, a significant burst activity remained until cycle $\mathrm{n}^{\circ} 10$, and few bursts randomly occurred until cycle $n^{\circ} 300$. The maximum plastic strain rate recorded at a given cycle slightly increased with increasing stress level. Otherwise, the plastic strain rate decreased as cycling progressed. $\varepsilon_{p l}$ rapidly decreased as the sample was cycled at a given stress step, becoming undetectable after approximately 4-5 cycles. This gradual diminution of $\dot{\varepsilon}_{p l}$ was observed for all the stress steps. However, at 3-30 MPa, the decrease was slower than for the other stress steps and plastic strain rate was recorded for 10 cycles.

Figure 6(b) presents the associated acoustic power. The same tendency observed for the stress amplitude of $1.5-15 \mathrm{MPa}$ was observed for all the different steps. Over one cycle, the AE power is strongly correlated with the plastic strain rate. However, the relation between $d E / d t$ and $\varepsilon_{p l}^{\cdot}$ changed with the cyclic step. For example, the continuous part of the AE power was similar regardless of the stress imposed (Figure 6(b)) for cycle $\mathrm{n}^{\circ} 2$, whereas the associated plastic strain rate continuously increased with the stress amplitude (Figure 6(a)). The maximum AE power decreased as cycling progressed. The first two steps (1-10 and $1.5-15 \mathrm{MPa}$ RSS) present similar evolution in terms of continuous and discrete AE, whereas a larger amount of discrete $\mathrm{AE}$ with high energy was observed for the last two steps at 2.5-25 and 3-30 MPa. After four cycles, the acoustic signal remained flat, indicating 
that plastic deformation no longer occurred in the sample anymore, except for the 3$30 \mathrm{MPa}$ stress step in which small fluctuations were detected until cycle $\mathrm{n}^{\circ} 10$.

The increase of $\varepsilon_{p l}$ and the associated $\Delta \varepsilon_{p l}$ (plastic strain increment over a cycle) are shown in Figure 6(c). $\Delta \varepsilon_{p l}$ was greater for cycle ${ }^{\circ} 1$ for the $1-10 \mathrm{MPa}$ step than for the next (1.5-15/2.5-25/3-30 MPa) stress steps. However, the increase of $\varepsilon_{p l}$ over the subsequent cycles was larger when increasing the imposed stress, as indicated by the increase of $\Delta \varepsilon_{p l}\left(e . g\right.$., cycle $\left.\mathrm{n}^{\circ} 2\right)$.

a)

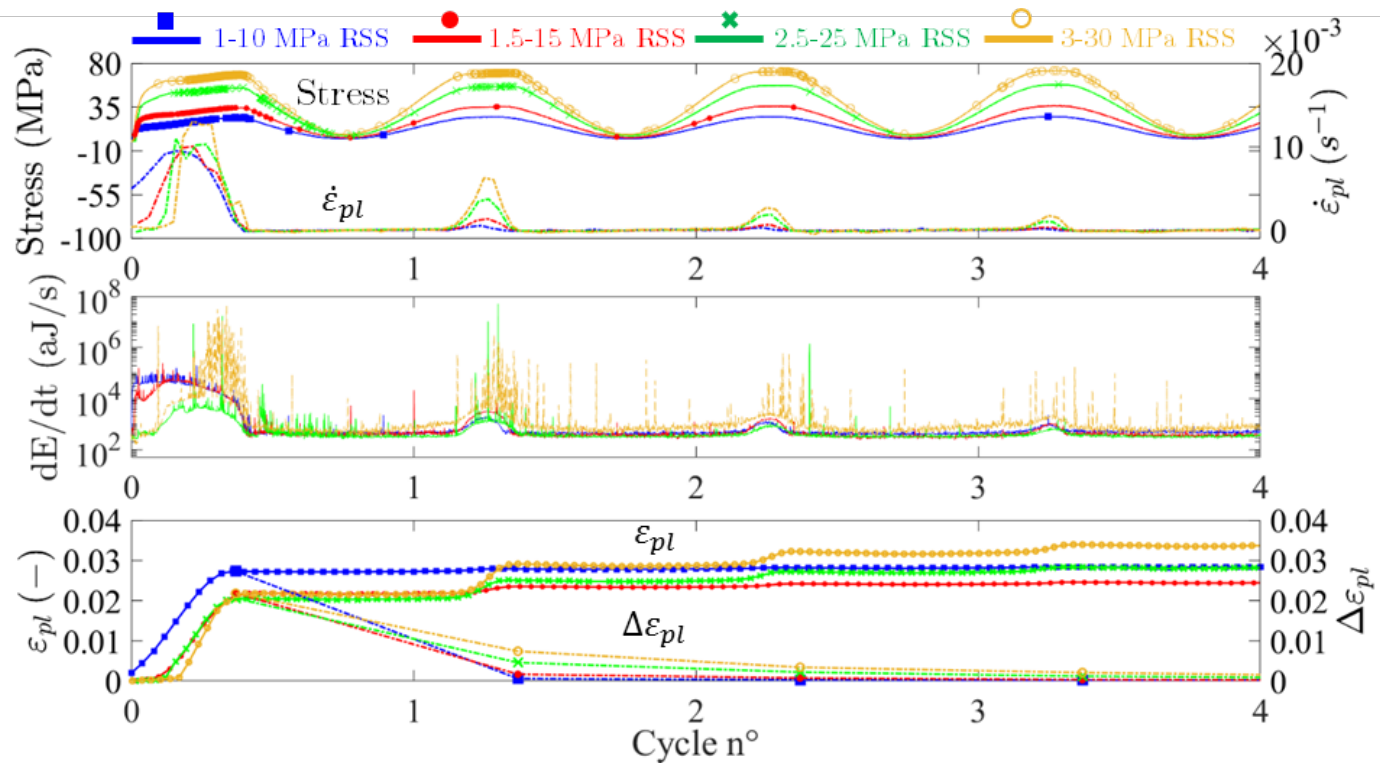

Figure 6. (a) Evolution of stress and plastic strain rate over four cycles. The colored dots indicate the stress levels at which AE bursts were detected. (b) Evolution of acoustic power. (c) Evolution of plastic strain $\boldsymbol{\varepsilon}_{\boldsymbol{p l}}$ and plastic strain amplitude $\Delta \boldsymbol{\varepsilon}_{\boldsymbol{p l}}$.The blue, red, green, and yellow colors refer to the 1-10, 1.5-15, 2.5-25, and 3-30 MPa RSS steps, respectively.

For all the stress steps, no further plastic strain was generated after a few cycles, as a result of rapid hardening. The largest $\Delta \varepsilon_{p l}$, most of the AE bursts, and the largest acoustic power were observed during the first cycle for all the stress steps, indicating that most of the deformation processes occurred during cycle $\mathrm{n}^{\circ} 1$. A gradual diminution of $\Delta \varepsilon_{p l}$, of the number of bursts, and of the AE power, was observed in the subsequent cycles for all the stress steps. These decreases were observed because the crystal progressively hardened enough to accommodate the imposed stress.

\subsubsection{Analysis of dislocation avalanches}

Dislocation avalanches sizes or energies have been shown to be power-law distributed $[9,11,49,50]$ over a certain lower cut-off $X_{\text {cutoff }}$,

$$
p(x) \propto x^{-\tau_{x}},
$$

where $x$ is the studied parameter and $\tau_{x}$ is a power-law exponent. Recently, this exponent was found to depend on the crystal structure [7], the sample size, the 
internal disorder (presence of solutes or precipitates)[50], or the cyclic strain hardening [10], and related with the wildness $W$, with large $W$ associated with smaller exponents [7,50]. Here we estimated, for the avalanche energy distributions, the exponent $\tau_{E}$ and the cut-off $E_{\text {cutoff }}$, as well as their uncertainties, using the maximum likelihood estimation (MLE) methodology proposed by Clauset et al. [51]. These energy distributions are shown in Figure 7, aggregating all the bursts detected during all the cycles of a given stress step.

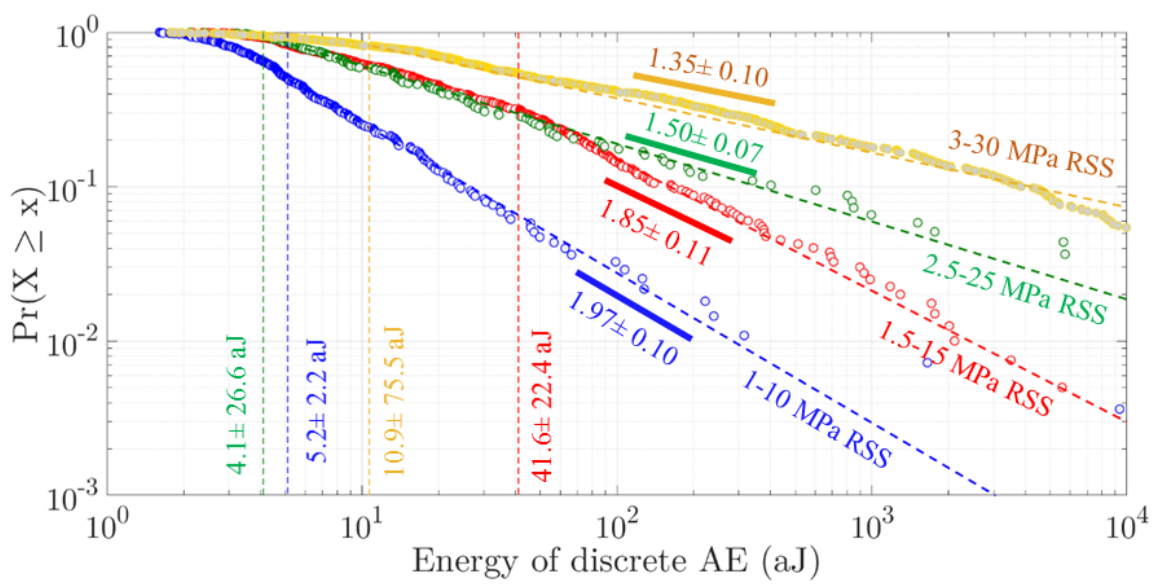

Figure 7. Cumulative probability density function of discrete AE energy (aggregating all the bursts detected during all the cycles of the cyclic step) for the different stress steps. The vertical dashed lines represent $E_{\text {cutoff }}$ for each step.

Upon increasing the stress amplitude from 1-10 $\mathrm{MPa}$ to 3-30 $\mathrm{MPa}$, the critical exponent $\tau_{E}$ decreased from 1.97 to 1.35 . These values fall within the commonly observed range $[14,52]$. A power-law distribution was observed above a cut-off value $E_{\text {cutoff }}$ which ranged from $4 \mathrm{aJ}$ (for the 1-10, 2.5-25, and 3-30 MPa steps) to $41 \mathrm{aJ}$ (1.5-15 MPa step). Power law scaling was observed in all cases over almost three decades. The decrease of $\tau_{E}$ indicates that a larger imposed stress amplitude results in a higher probability of observing high-energy bursts. The investigation of the evolution of these power laws with cycling, within a given stress step, has been restricted by the limited number of $\mathrm{AE}$ bursts recorded.

Brown \& al. [53,54] propose to model an avalanche of dislocations as the displacement of an ellipsoidal gliding band, whose size is power-law distributed. The average size of the ellipsoidal band is inversely proportional to the applied stress. This is supported by the fact that the more the stress increases, the larger the dislocation density is around the avalanche, hindering its motion (local activation of cross-slip and activation of new slip systems). Depending on the loading mode and the hardening rate, the critical exponent of the power-law ranges between 1.5 (in case of stage II monotonic deformation) and 2 in the case of cyclic fatigue. The authors suggest that these exponents are representative of the system's ability to block avalanches. In the case of $\tau=1.5$, avalanches are supposed to be stopped by the activation of the local secondary slip and in the case of $\tau=2$, by structural features such as cross-slip or grain boundaries. In the present case, we observe a range of 
exponents slightly wider than 1.5-2. Moreover, the more the stress increases, the more energetic avalanches are observed (decrease of $\tau_{E}$ with increased stress), which means that these avalanches can travel long distances without being stopped by the local environment.

Finally, the evolution of $W_{A E}$ with cycling was studied for the different loading steps. Figure 8 shows the evolution of $W_{A E}$ (left subplot) and $\Delta \varepsilon_{p l}$ (right subplot) over the first ten cycles as a function of the imposed stress. AE bursts with energies below $E_{\text {cutoff }}$ were not considered in the calculation of $W_{A E}$ because they were not considered as a signature of wild dislocation avalanches [50]. The error bars for the values of $W_{A E}$ are related to the uncertainties of the $E_{\text {cutoff }}$ values. Overall, $\mathrm{W}_{\mathrm{AE}}$ increased upon increasing the imposed stress from 1-10 MPa to 3-30 MPa, and it remained significant over a larger number of cycles during the last stress steps.

For all the stress steps, the plastic strain amplitude was always the greatest during cycle $\mathrm{n}^{\circ} 1$ and then continuously decreased with increasing cycle number until reaching a value close to zero. For a larger imposed stress, more cycles were required before reaching the fully hardened state. The evolution of $W_{A E}$ was similar for cyclic steps at 1-10 and 1.5-15 MPa, for which the wildness was maximum in the first cycle and then rapidly decreased with increasing cycle number (blue $\mathbf{\square}$ and red $\bullet$ curves of Figure 8 ). The wildness of cycle $\mathrm{n}^{\circ} 1$ increased from $\sim 4 \%$ to $\sim 86 \%$ upon increasing the imposed stress from 1-10 MPa to 3-30 MPa. The associated $\Delta \varepsilon_{p l}$ value during cycle $\mathrm{n}^{\circ} 1$ is quite independent of the imposed stress level. The decrease of $\Delta \varepsilon_{p l}$ along cycles is quite similar to the evolution of $W_{A E}$. Upon increasing stress levels, $W_{A E}$ greatly increases, especially during the very first cycle and wildness remains for more cycles.
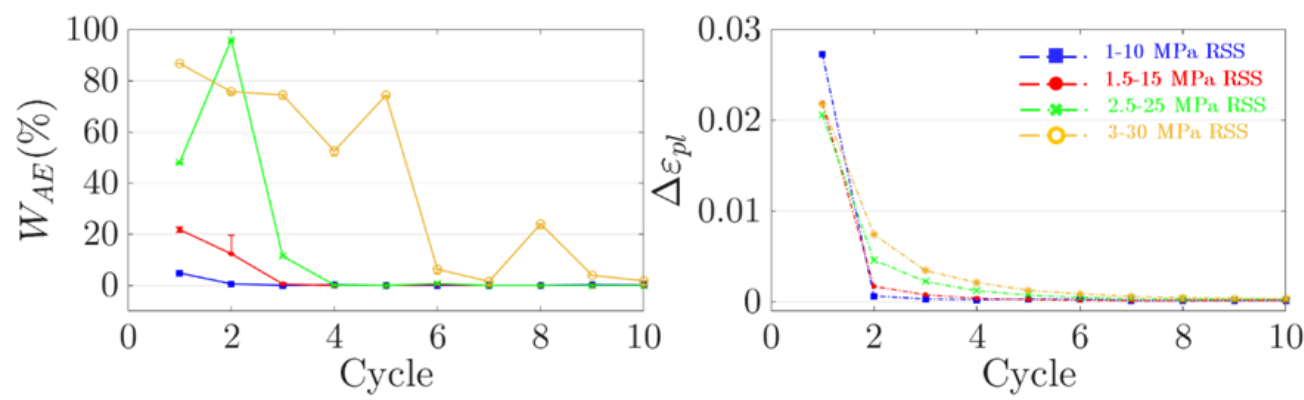

Figure 8. Evolution of $W_{A E}(\%)$ and $\Delta \boldsymbol{\varepsilon}_{p l}$ during the first ten cycles of each cyclic step.

\subsection{Microstructural characterization}

EBSD maps (texture component) and R-ECCI images showing the evolution of the dislocation structure with imposed stress from the initial state to the 3-30 MPa RSS step are presented in Figure 9. The texture component gives information about the orientation difference with respect to a reference orientation (chosen in each image). Dislocation mean free paths, which are supposed here to corresponds to the characteristic length of the dislocation structure (cell size, and distance between walls), were measured manually. The mean free path $\lambda\left(\lambda_{c}\right.$ and $\lambda_{w}$ referred to the 
mean path of the cell and the wall structure, respectively) related to each dislocation structure is indicated on the right of Figure 9.

The dislocation density $\rho$ has been estimated using the same methodology as the one proposed in Ham works [55]. The intercept method was used to count the number of dislocations crossing a grid consisting of 7 lines. The dislocation density $\rho$ is defined as $\rho=2 N / l t$, where $N$ is the number of counted dislocations and $l$ is the total grid length. The number 2 is a correction factor that considers the stereographic projection of dislocations. The probe depth $t$ was assumed to be $\sim 100 \mathrm{~nm}$, as described in [41]. High magnification images were used to count dislocations. These densities remain approximated because we do not know precisely the analyzed depth, and dislocation density has not been estimated from several different diffraction vectors g. Moreover, when the dislocation structure becomes very dense, it becomes difficult to count dislocations correctly because some of them can no longer be discriminated. Thus, the given dislocation densities are lower bound densities, but they can be compared to each other's. All the dislocation densities are indicated on the right of Figure 9.

The as-received copper single crystal exhibited a disorientation of less than $2^{\circ}$ with mean disorientation of approximately $0.4^{\circ}$ (Figure 9(a)). The dislocation structure was composed of circular cells (Figure $9(\mathrm{~g}, \mathrm{~m})$ ) with a mean cell size of $0.9 \pm 0.4 \mu \mathrm{m}$. The dislocation density in this initial state was estimated to be $\sim 3.4 \pm$ $0.4 * 10^{13} \mathrm{~m}^{-2}$.

The initial dislocation structure was unexpected for an undeformed specimen, as numerous organized dislocation cells were present. However, the cell walls consisted of only 3-4 dislocations and were not considered as dense as the dislocation structure as formed after cyclic deformation, where dislocations could no longer be individually discriminated [56]. Moreover, this structure was most likely formed at the surface of the specimen during the elaboration process. It is likely that the first polishing did not remove the entire perturbed layer.

The five cyclic steps at RSS of 1-10, 1.5-15, 2-20, 2.5-25, and 3-30 MPa resulted in cumulated cyclic plastic strains of $2.9 \%, 5.5 \%, 7.7 \%, 10.8 \%$, and $14.6 \%$. The texture components showed a gradual increase of disorientation with the strain (Figure 9(a-f)).

After the 1-10 MPa step, the mean disorientation is about $1^{\circ}$ (with a maximum of less than $3^{\circ}$ ). Disorientation bands (DBs) appeared parallel to the $(\overline{111})$ and $(\overline{2} 1 \overline{2})$ planes (Figure $9(\mathrm{~b}))$. In this state, large and equiaxed dislocation cells were observed with a mean cell size of $2.7 \pm 0.3 \mu \mathrm{m}$ and a cell wall width of $0.35 \pm 0.12$ $\mu \mathrm{m}$ (Figure 9(h,n)). In this state, the dislocation density is about $\sim 1.5 \pm 0.2 *$ $10^{13} \mathrm{~m}^{-2}$.

After cyclic loading at 1.5-15 $\mathrm{MPa}$, the mean disorientation increased to $1.3^{\circ}$, with local maximum values of up to $\sim 4^{\circ}$ in the developing DBs (Figure $9(\mathrm{c})$ ). In this state, the formation of a wall structure was observed. The width of these walls was $0.24 \pm 0.07 \mu \mathrm{m}$, and the distance between walls was $2.8 \pm 1.3 \mu \mathrm{m}$. This structure formed 
inside the DBs, whereas a cell structure was present outside the DBs (Figure 9(i,o)), with cell walls width of $0.18 \pm 0.06 \mu \mathrm{m}$ and a mean cell size of $1.9 \pm 0.5 \mu \mathrm{m}$. An increase in the area covered by DBs was observed for the 2-20 MPa level (Figure $9(\mathrm{~d})$ ). The mean disorientation was $\sim 7.3^{\circ}$. The DBs appeared to rotate until becoming parallel to $(\overline{111}),(\overline{1} 1 \overline{2})$, and $(\overline{1} 1 \overline{1})$ planes, which has been observed elsewhere [57]. The presence of the wall structure in the DBs was confirmed by ECCI (Figure $9(\mathrm{j}, \mathrm{p})$ ). The walls were thinner $(0.22 \pm 0.06 \mu \mathrm{m})$ but the channels between them were narrower $(2.3 \pm 0.9 \mu \mathrm{m})$ compared with those for the previous stress level. The mean cell size of the cell structure was $1.9 \pm 0.4 \mu \mathrm{m}$ with a cell walls width of $0.15 \pm 0.03 \mu \mathrm{m}$.

After the 2.5-25 MPa step, DBs with different disorientations covered the studied area, with a maximum disorientation of $16^{\circ}$ (Figure 9(e)). In addition, both cell and wall structures built up. The cell size (approximately $1.2 \pm 0.3 \mu \mathrm{m}$ ) and distance between walls $(1.6 \pm 0.6 \mu \mathrm{m})$ were smaller than those for the previous steps (Figure $9(\mathrm{k}, \mathrm{q})$ ). In the final cyclic step, the wall structure became very dense, with a wall width of $0.18 \pm 0.09 \mu \mathrm{m}$ and a mean distance between walls of $1.3 \pm 0.5 \mu \mathrm{m}$. The cell structure did not change substantially compared with that of the previous stress step. However, a build-up of the cell structure was observed as the cell size is reduced down to $1.0 \pm 0.3 \mu \mathrm{m}$. Maximum disorientation of $\sim 19^{\circ}$ was estimated from the EBSD analysis. Upon increasing stress amplitude, dislocation density increases and the dislocation mean free path (in both cells and walls) decreases.

The gradual increase of the global disorientation and the formation of the disorientation bands are attributed to lattice rotation during cyclic loading. Because of slip activity, the crystal lattice rotated from the [110] to the [100]-[111] boundary of the stereographic triangle with local rotations of the crystal lattice along this boundary toward the [211] direction [56]. 

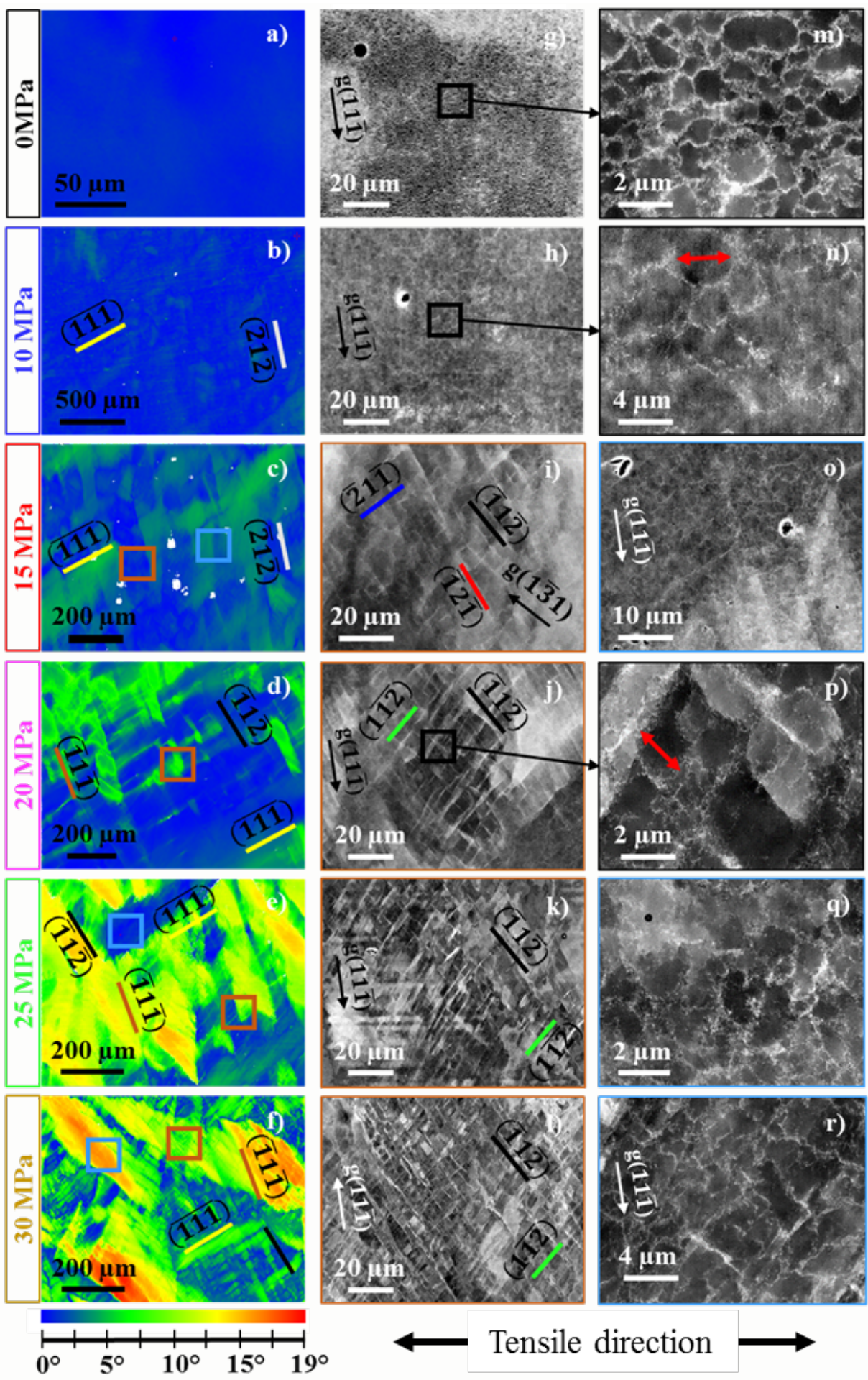

$\lambda_{c}$ : cell structure

$\lambda_{w}$ : wall structure

$\rho_{c}$ : cell dislocation density

$\rho_{c}$ : wall dislocation density

$\lambda_{c}=0.9 \pm 0.4 \mu \mathrm{m}$

$\rho_{c}=\sim 3.4 \pm 0.4 * 10^{13} \mathrm{~m}^{-2}$

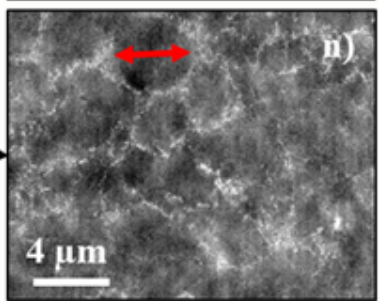

$\lambda_{c}=2.7 \pm 0.3 \mu \mathrm{m}$
$\rho_{c}=\sim 1.5 \pm 0.2 * 10^{13} \mathrm{~m}^{-2}$

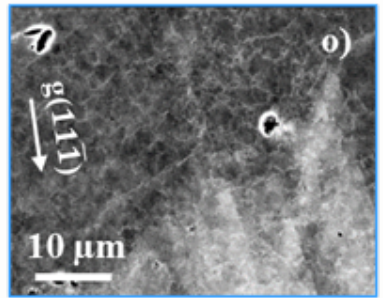

$\lambda_{c}=1.9 \pm 0.5 \mu \mathrm{m}$

$\lambda_{w}=2.8 \pm 1.3 \mu \mathrm{m}$

$\rho_{c}=\sim 3.0 \pm 0.3 * 10^{13} \mathrm{~m}^{-2}$

$\rho_{w}=\sim 2.2 \pm 0.3 * 10^{13} \mathrm{~m}^{-2}$

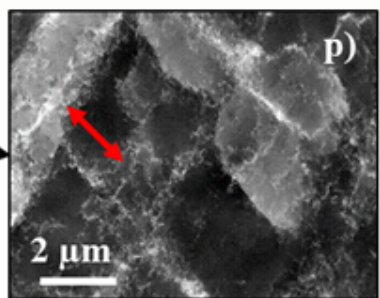

$\lambda_{c}=1.9 \pm 0.4 \mu \mathrm{m}$

$\lambda_{w}=2.3 \pm 0.9 \mu \mathrm{m}$

$\rho_{c}=\sim 3.1 \pm 0.2 * 10^{13} \mathrm{~m}^{-2}$

$\rho_{w}=\sim 3.4 \pm 0.3 * 10^{13} \mathrm{~m}^{-2}$

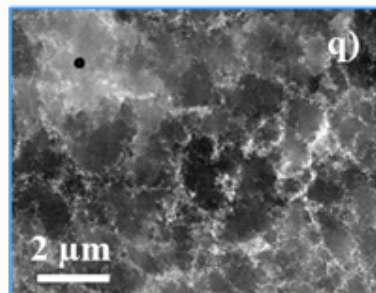

$\lambda_{c}=1.2 \pm 0.3 \mu \mathrm{m}$

$\lambda_{w}=1.6 \pm 0.6 \mu \mathrm{m}$

$\rho_{c}=\sim 3.3 \pm 0.2 * 10^{13} \mathrm{~m}^{-2}$

$\rho_{w}=\sim 3.7 \pm 0.3 * 10^{13} \mathrm{~m}^{-2}$

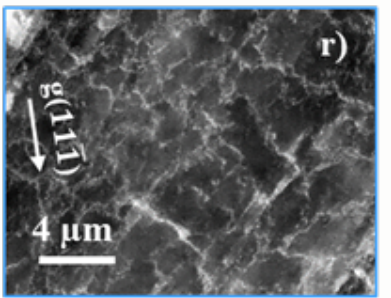

$\lambda_{c}=1.2 \pm 0.4 \mu \mathrm{m}$

$\lambda_{w}=1.3 \pm 0.5 \mu \mathrm{m}$

$\rho_{c}=\sim 3.5 \pm 0.3 * 10^{13} \mathrm{~m}^{-2}$

$\rho_{w}=\sim 4.8 \pm 0.4 * 10^{13} \mathrm{~m}^{-2}$

\section{Tensile direction}

Figure 9. Microstructure characterization of the loading steps. EBSD maps are shown in the left-hand column. Dislocations appear in white in the ECC-Images. The measured dislocation mean free path and dislocation densities are indicated on the right. Texture component from EBSD maps (a-f). Dislocation structure of as-received state ( $g, m)$. Dislocation structures for stress amplitudes of 1-10 MPa (h,n), 1.5-15 MPa (i,o), 2-20 MPa (j,p), 2.5-25 MPa $(k, q), 3-30 \mathrm{MPa}(l, r)$. Red arrows show how $\lambda$ were measured. 


\section{Discussion}

\subsection{Dislocation structure and mechanical behavior}

As seen in part 3.3, each stress amplitude increment leads to the formation of a new dislocation structure, with different characteristic arrangements and dislocation mean free paths. This implies that such incremental stress induces a profound rearrangement of the microstructure. During a fatigue test with a constant imposed stress amplitude (regardless of the applied stress), a strong initial hardening is observed but quickly vanishes: after about 10 cycles, the deformation is almost entirely elastic. Thus, the gradually generated dislocation structure is only able to accommodate the imposed stress amplitude and must necessarily rearrange if the stress amplitude increases.

The loading mode and the control mode play an important role in the evolution of the dislocation structure. The stress levels from the last two fatigue steps (2.5-25 and 3-30 MPa RSS) are of the order of those required for the formation of persistent slip bands (PSB) during stress-imposed cyclic loading [29,30], which are not observed in our case. PSB are formed under specific conditions [32]: the mean stress must be null or near zero and the plastic strain amplitude, must be in the range of $10^{-4} \leq$ $\Delta \varepsilon_{p l} \leq 10^{-2}$. During our stress steps, the mean stress is always higher than zero, due to the loading ratio $(R \sigma=0.1)$, which explains why no PSB are observed. The control mode also influences the dislocation structure formation. During strain-controlled cyclic loading, the crystal is deformed at every cycle and cyclic hardening, followed by cyclic softening (in some cases) and saturation of the cyclic stress amplitude are observed $[19,20,56]$.

In our stress-controlled tests, only cyclic strain hardening followed by saturation is observed. Under this loading mode, the saturation stage is characterized by a vanishing plastic strain amplitude. The cyclic hardening is due to the rapid multiplication and the subsequent entanglement of dislocations. This implies that the reorganizations of the dislocation structure take place within few cycles at the beginning of the cyclic stress steps. Thus, the question of how these rearrangements occur arises.

\subsection{Dislocation dynamics evolution at a given stress amplitude and with increasing stress}

For a given imposed stress level, a dislocation structure quickly forms and becomes more stable with increasing cycling. Strain hardening ensues, which is expressed, in this stress imposed mode $\left(R_{\sigma}=0.1\right)$, by a progressive fading of plastic deformation over the cycles (Figure 7(b)). This is directly related to a decrease in AE signals (both continuous and discrete) over the cycles.

The decrease of the maximum of continuous $\mathrm{AE}$ power with cycling is directly related with a simultaneous decrease of plastic deformation. Over the very first cycles, both the discrete AE activity (number of detected bursts/cycle) and the wildness $W_{A E}$ (proportion of $\mathrm{AE}$ energy accounted by $\mathrm{AE}$ bursts) decrease very 
rapidly through cycling (Figure 5 and Figure 8). This strongly suggests that the reorganization of the dislocation structure occurs in an intermittent manner, through dislocation avalanches spreading over distances much larger than the mean free path inherited from the previous loading step, as no upper cut-off is detected on the power law distributions of $\mathrm{AE}$ energies (Figure 7). As the new dislocation structure gets more stable, the wildness vanishes. The prominent existence of dislocation avalanches during the first cycles was also observed by Hong and Laird [37]. They observed a rapid fading of avalanches (after approximately 5 cycles) and hypothesized that the crystal was rapidly hardened, leading to no further deformation after 5 cycles.

Regardless of the imposed stress amplitude, strain hardening quickly fades after few cycles (4-5 cycles for stress amplitude of 1-10/1.5-15 and 2.5-25 MPa and $\sim 10$ cycles for $3-30 \mathrm{MPa})$. The evolution of the continuous acoustic power through cycling is similar for all the stress steps.

The number of bursts increases with the stress level. However, they remain confined to the first cycles (except for the cyclic stress step of 3-30MPa for which dislocation avalanches are recorded for a larger number of cycles). Laird et al.[35] made a qualitatively similar observation on polycrystalline copper during cyclic loading. The part of plasticity accommodated through dislocation avalanches, $W_{A E}$, increases when the stress amplitude increases (Figure 8). This is consistent with a decrease of the critical exponent value $\tau_{E}$ of the power law distributions (Figure 7), as an anti-correlation between the exponent and the wildness has been recognized previously $[12,50]$. This indicates that high-energy AE bursts are more likely to be generated upon increasing stress level and increasing complexity of the structure. In the case of the 3-30MPa step, a slower decrease of the wildness with cycling is observed (Figure 8). After four cycles, although plastic deformation almost vanished, the wildness remains important. This suggests that few brutal rearrangements can still take place within the nascent dislocation structure.

\subsection{Acoustic emission signals and dislocation structure}

4.3.1. Mean free path and continuous acoustic power

The continuous part of the acoustic power $d E_{c} / d t$ is considered to originate from plastic deformation resulting from a cumulative effect of numerous, small and uncorrelated dislocation motions corresponding to a mild plasticity. Many authors observed a link between the acoustic power and the plastic strain rate. Rouby et al.[58] and others $[38,59,7]$ linked the continuous part of the acoustic power $d E_{c} / d t$ to the plastic strain rate $d \varepsilon_{p l} / d t$ from a simple model:

$$
\frac{d E_{c}}{d t} \sim b \bar{s} \frac{d \varepsilon_{p l}}{d t}
$$

where $b$ is the Burger's vector and $\bar{s}$ is the mean value of the individual dislocation sweeping area. It is assumed to be linked to the dislocation mean free path $\lambda$ characterizing the dislocation structure through $\lambda \sim \sqrt{\bar{s}}$. However, so far, no clear 
data has ever been presented to validate this hypothesis. The value of $\bar{s}$ can be extracted from eq. 2 as follows:

$$
\bar{S} \sim \frac{d E_{c} / d t}{b d \varepsilon_{p l} / d t} .
$$

Figure 10(a) shows the evolution of $d \varepsilon_{p l} / d t$ and $d E_{c} / d t$ during cycles 1 and 2 at 1.5-15 MPa. As both $d \varepsilon_{p l} / d t$ and $d E_{c} / d t$ were independently evaluated in these experiments, $\bar{s}$ can be estimated. Since the time resolution of the AE and DIC methods are not the same (AE: $10 \mathrm{~ms}$; DIC: $0.3 \mathrm{~s}$ ), a linear interpolation of the plastic strain rate was performed. The continuous part of the $\mathrm{AE}$ power $d E_{c} / d t$ is extracted from the recorded $\mathrm{AE}$ power by carefully removing the instrumental background noise as well as the bursts corresponding to dislocation avalanches. For each cycle, $\bar{s}$ is estimated as the best-fit between $d \varepsilon_{p l} / d t$ and $d E_{c} / d t$.

To validate the relationship between $\bar{S}$ and $\lambda, \bar{S}$ was compared with the dislocation mean free path estimated from ECC image analysis (mean cell size, mean distance between walls) for all the stress steps (Figure 10(b)). Considering that the dislocation structure is composed of both cells and walls from 1.5-15 to 3-30 MPa, for which the mean free paths are not the same, an average mean free path $\lambda_{a v^{\circ}}$ was used. The fraction of the structure containing cells or walls could not be precisely estimated. We made the assumption of a 50\% fraction of both walls and cells regardless of the stress step (except at 1-10 $\mathrm{MPa}$, for which only cells were observed), which enabled an average mean free path $\lambda_{a v^{\circ}}$ to be defined for each stress step. The value of $\bar{s}$ at cycle 1 was compared with the average mean free path of the dislocation structure observed just before, i.e. after the last cycle of the previous stress step. For example, the value of $\bar{s}$ at cycle 1 at $2.5-25 \mathrm{MPa}$ was compared with $\lambda_{a v^{\circ}}$ of the dislocation structure generated after 5000 cycles at 2-20 MPa. We chose to compare $\sqrt{\bar{S}}$, generated during the first cycle of each stress step (cycle where the main plastic phenomena occur), to the dislocation structure previously created. Since $\sqrt{\bar{S}}$ is theoretically related to the characteristic sizes of the dislocation structure, it must be constrained by the dislocation structure obtained at the end of the previous stress step. Figure 10(b) shows the evolution of $\sqrt{\bar{S}}$ (to be consistent with a length rather than an area) and of the average mean free path with $\mathbf{1} / \sqrt{\boldsymbol{\rho}}$. A similar evolution of $\sqrt{\bar{s}}$ and $\lambda_{\mathrm{av}}{ }^{\circ}$ can be observed from this figure, which validates the model previously proposed by Rouby et al.[58] and others [38,59,7]. The scale of the dislocation structure can thus be characterized using three different methods. Indeed, the decrease of the mean free path estimated from ECC-images is consistent with the increase of $\rho$ (from ECCI) as well as with the decrease of $\bar{s}$ determined from AE and DIC measurements.

Figure 10(c) shows the evolution of $\bar{s}$ over the first four cycles for each stress step. Regardless of the stress amplitude, $\bar{s}$ decreased as cycling progressed. This implies that the emergence of a new dislocation structure is accompanied by a decrease of the dislocations' mean free path. In addition, for cycle $n^{\circ} 1$ of each cyclic step, the value of $\bar{s}$ decreased with increasing stress. 


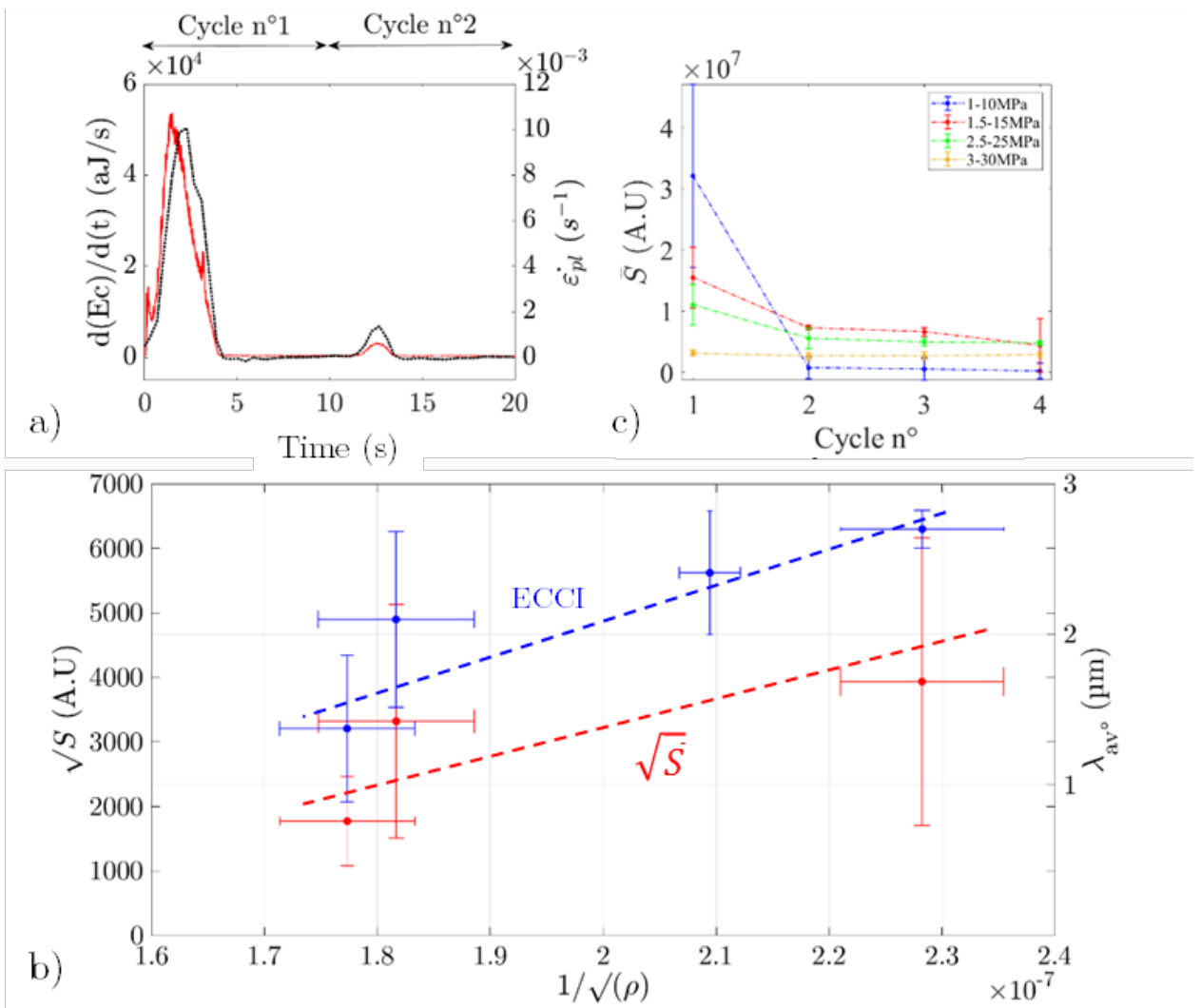

Figure 10. (a) Relation between $\boldsymbol{\varepsilon}_{\boldsymbol{p l}}$ and $\boldsymbol{d} \boldsymbol{E}_{\boldsymbol{c}} / \boldsymbol{d} \boldsymbol{t}$ during the two first cycles of the 1.5-15MPa step. (b) Evolution of $\sqrt{\overline{\boldsymbol{s}}}$ and $\boldsymbol{\lambda}_{\boldsymbol{a} v^{\circ}}$ from ECCI with $\mathbf{1} / \sqrt{\boldsymbol{\rho}}$ (c) Evolution of $\overline{\boldsymbol{s}}$ for the first four cycles of each cyclic step.

\subsubsection{Dislocation dynamics}

Upon increasing stress level, the initial cell structure is converted into a complex structure made of cells, walls, and DBs coexisting together. Dislocation cells size and distance between walls gradually reduce, while the dislocation density increases. DBs, first formed during the $1.5-15 \mathrm{MPa}$ cyclic stress step, are present in an increasing volume of the specimen when the stress is increased. Thus, the dislocation structure is denser as the stress is increased.

We have shown in part 4.3.1, that during the first fatigue cycle, the continuous part of the acoustic power (mild plasticity) keeps the memory of the dislocation mean free path of the previous microstructural state. This is illustrated by the correlation between the values of $\sqrt{\bar{s}}$ and $\lambda_{\mathrm{av}^{\circ}}$ Figure 10(b)). At the same time, dislocation avalanches are numerous during those first cycles. These dislocation avalanches are the signature of profound reorganizations of dislocation structures, implying a rapid vanishing of this memory. Through cycling within a given stress step, the mean free path decreases (decrease of $\bar{s}$ ) as a new dislocation structure emerges. Simultaneously, the dislocation avalanche activity decreases, as illustrated by a decrease in the number of avalanches and of the wildness with increasing number of cycles. This decrease of $W_{A E}$ with a decrease of the mean free path is fully consistent with the study of Zhang $\&$ al. [50]. In their study of plastic instabilities in nanopillars, they showed that the 
characteristic internal length scale of the microstructure plays a crucial role on the wildness of plasticity. In their case, this internal length scale is set by the nature of the alloy, through the density and strength of the obstacles to dislocation motions (solutes, precipitates). In the case described here (pure metals), the internal scale naturally emerges from dislocation short-range interactions and corresponds to the mean free path of the dislocation structure.

However, we note that the number and the average energy of dislocation avalanches increase with the increase of the stress amplitude. This may seem counterintuitive at first glance because when the stress is increased, the dislocation density increases and the dislocation mean free path decreases. However, this could result from the combination of both an increase of elastic energy brought to the crystal upon increasing stress, and the break-up of walls or cells containing a larger dislocation density.

The idea that a dislocation structure may collapse in the form of dislocation avalanches during imposed stress fatigue loading was also proposed by P.Neumann (stress ramp on $\mathrm{Cu}$ single crystals) [33,34] and C.Laird (constant stress amplitude) $[35,60]$. In both cases, the authors observe strain bursts during cyclic loading and hypothesize that these strain bursts are the consequence of a global collapse of the dislocation structure, in the form of dislocation avalanches gradually spreading throughout the crystal. If dislocation avalanches in our case have the same origin, we do not observe these macroscopic strain bursts. In our study, avalanches are contained within the cycles and we show that they coexist with a mild plasticity, which is not discussed in the work of Neumann and Laird.

In other words, the reorganization of a denser dislocation structure occurs through more energetic dynamical events. If the concept of dislocation mean free path seems adequate to explain the mild plasticity, questions can be raised about its pertinence and meaning in the case of dislocation avalanches.

\section{Conclusion}

Plasticity dynamics can take place in the form of numerous but small and uncorrelated dislocation motions (mild plasticity), in the form of synchronous dislocation motions named dislocation avalanches (wild plasticity), or through a combination of both. In this work, the correlation between microstructural evolutions and dislocation patterning under cyclic loading on the one hand, and the nature of collective dislocation dynamics on the other hand was studied. Stress-imposed cyclic loadings on the very same [110] copper single crystal specimen were performed with increasing stress amplitudes. Dislocation dynamics was studied through $\mathrm{AE}$ measurements and dislocation structures were characterized before and after each cyclic loading using EBSD and ECCI.

During cyclic loading under constant imposed stress amplitude, plastic strain mostly occur during the very first cycles. Cyclic strain hardening, resulting from the emergence of a dislocation structure is observed through cycles and is accompanied by a decrease of mild plasticity (together with a decrease of the dislocation mean free 
path, observed by both ECCI and AE, through $\sqrt{\bar{s}}$ ), a decrease in the number of dislocation avalanches and a decrease of the wildness of plasticity.

Upon increasing the stress level, this dislocation structure is modified. The initial cell structure gives way to the formation of two coexisting structures: one made of dense walls (inside disorientation bands) and one consisting of dense cells. These microstructural evolutions show that, as soon as the stress is increased, the former dislocation structure is no longer stable and undergoes major rearrangements. Those reorganizations occurred at the beginning of the cyclic steps and took place, at least partly, through dislocation avalanches. We noticed that the larger the imposed stress amplitude, the more energetic the plastic instabilities, whereas the dislocation mean free path decreases. This evolution indicates that, upon reloading at a larger stress amplitude, the dislocation structure is reorganized and dislocation avalanches can travel over distances much larger than the former mean free path. This illustrates the complex interplay between dislocation avalanches and the emergence of a stable dislocation structure.

\section{Acknowledgments}

This research did not receive any specific grant from funding agencies in the public, commercial, or not-for-profit sectors. The authors would like to acknowledge CLYM (www.clym.fr) for access to the NVISION microscope.

\section{Declaration of interest}

Declaration of interest - none

\section{References}

[1] F. Barlat, A simple model for dislocation behavior, strain and strain rate hardening evolution in deforming aluminum alloys, International Journal of Plasticity. 18 (2002) 919-939. doi:10.1016/S0749-6419(01)00015-8.

[2] I. Gutierrez-Urrutia, S. Zaefferer, D. Raabe, Electron channeling contrast imaging of twins and dislocations in twinning-induced plasticity steels under controlled diffraction conditions in a scanning electron microscope, Scripta Materialia. 61 (2009) 737-740. doi:10.1016/j.scriptamat.2009.06.018.

[3] R. Madec, B. Devincre, L.P. Kubin, From Dislocation Junctions to Forest Hardening, Physical Review Letters. 89 (2002) 255508-255508. doi:10.1103/PhysRevLett.89.255508.

[4] B. Devincre, T. Hoc, L. Kubin, Dislocation Mean Free Paths and Strain Hardening of Crystals, Science. 320 (2008) 1745-1748. doi:10.1126/science.1156101.

[5] M. Sauzay, L.P. Kubin, Scaling laws for dislocation microstructures in monotonic and cyclic deformation of fcc metals, Progress in Materials Science. 56 (2011) 725-784. doi:10.1016/j.pmatsci.2011.01.006.

[6] Y. Bergstrom, A Dislocation Model for the Stress-Strain Behaviour of Polycrystalline a-Fe with Special Emphasis on the Variation of the Densities of Mobile and Immobile Dislocations, Materials Science and Engineering. 5 (1970) 193-200.

[7] J. Weiss, W.B. Rhouma, T. Richeton, S. Dechanel, F. Louchet, L. Truskinovsky, From Mild to Wild Fluctuations in Crystal Plasticity, Physical Review Letters. 114 (2015). doi:10.1103/PhysRevLett.114.105504. 
[8] J. Weiss, J.-R. Grasso, Acoustic Emission in Single Crystals of Ice, The Journal of Physical Chemistry B. 101 (1997) 6113-6117. doi:10.1021/jp963157f.

[9] M.-C. Miguel, A. Vespignani, S. Zapperi, J. Weiss, J.-R. Grasso, Intermittent dislocation flow in viscoplastic deformation, Nature. 410 (2001) 667-671. doi:10.1038/35070524.

[10] M. Zaiser, Scale invariance in plastic flow of crystalline solids, Advances in Physics. 55 (2006) 185-245. doi:10.1080/00018730600583514.

[11] J. Weiss, T. Richeton, F. Louchet, F. Chmelik, P. Dobron, D. Entemeyer, M. Lebyodkin, T. Lebedkina, C. Fressengeas, R.J. McDonald, Evidence for universal intermittent crystal plasticity from acoustic emission and high-resolution extensometry experiments, Physical Review B. 76 (2007). doi:10.1103/PhysRevB.76.224110.

[12] J. Weiss, W. Ben Rhouma, S. Deschanel, L. Truskinovsky, Plastic intermittency during cyclic loading: From dislocation patterning to microcrack initiation, Physical Review Materials. 3 (2019). doi:10.1103/PhysRevMaterials.3.023603.

[13] J. Weiss, Ice: the paradigm of wild plasticity, Phil. Trans. R. Soc. A. 377 (2019) 20180260. doi:10.1098/rsta.2018.0260.

[14] T. Richeton, P. Dobron, F. Chmelik, J. Weiss, F. Louchet, On the critical character of plasticity in metallic single crystals, Materials Science and Engineering: A. 424 (2006) 190-195. doi:10.1016/j.msea.2006.03.072.

[15] O.U. Salman, L. Truskinovsky, Minimal Integer Automaton behind Crystal Plasticity, Physical Review Letters. 106 (2011). doi:10.1103/PhysRevLett.106.175503.

[16] S.J. Basinski, Z.S. Basinski, A. Howie, Early stages of fatigue in copper single crystals, Philosophical Magazine. 19 (1969) 899-924. doi:10.1080/14786436908225856.

[17] J.R. Hancock, J.C. Grosskreutz, Mechanisms of fatigue hardening in copper single crystals, Acta Metallurgica. 17 (1969) 77-97.

[18] A. Abel, Fatigue of copper single crystals at low constant plastic strain amplitudes, Materials Science and Engineering. 36 (1978) 117-124. doi:10.1016/0025-5416(78)90201-X.

[19] H. Mughrabi, The cyclic hardening and saturation behaviour of copper single crystals, Materials Science and Engineering. 33 (1978) 207-223.

[20] T. Tabata, H. Fujita, M.-A. Hiraoka, K. Onishi, Dislocation behaviour and the formation of persistent slip bands in fatigued copper single crystals observed by high-voltage electron microscopy, Philosophical Magazine A. 47 (1983) 841-857. doi:10.1080/01418618308243124.

[21] F. Ackermann, L.P. Kubin, J. Lepinoux, H. Mughrabi, The dependence of dislocation microstructure on plastic strain amplitude in cyclically strained copper single crystals, Acta Metallurgica. 32 (1984) 715-725. doi:10.1016/0001-6160(84)90145-7.

[22] M. Bao-Tong, C. Laird, Overview of fatigue behavior in copper single crystals-I. Surface morphology and stage I crack initiation sites for tests at constant strain amplitude, Acta Metallurgica. 37 (1989) 325-336. doi:10.1016/0001-6160(89)90217-4.

[23] B. Gong, Z.G. Wang, Y.W. Zhang, The cyclic deformation behavior of Cu single crystal oriented for double slip, A. 194 (1995) 171-178.

[24] R. Wang, H. Mughrabi, Secondary cyclic hardening in fatigued copper monocrystals and polycrystals, Materials Science and Engineering. 63 (1984) 147-163.

[25] T. Broom, R.K. Ham, The hardening of copper single crystals by fatigue, Proc. R. Soc. Lond. A. 251 (1959) 186-199. doi:10.1098/rspa.1959.0101.

[26] P.O. Kettunen, Fatigue hardening of copper single crystals at low stress amplitudes, Acta Metallurgica. 15 (1967) 1275-1286. doi:10.1016/0001-6160(67)90003-X.

[27] W.N. Roberts, Persistent slip bands in fatigued copper, Philosophical Magazine. 20 (1969) 675-686. doi:10.1080/14786436908228036.

[28] T.K. Lepistö, P.O. Kettunen, Comparison of the cyclic stress-strain behaviour of single-and 〈111〉 multiple-slip-oriented copper single crystals, Materials Science and Engineering. 83 (1986) 1-15. doi:10.1016/0025-5416(86)90169-2. 
[29] D. Melisova', B. Weiss', R. Stickle, NUCLEATION OF PERSISTENT SLIP BANDS IN Cu SINGLE CRYSTALS UNDER STRFSS CONTROLLED CYCLING, Scripta Materialia. 36 (1997) 6.

[30] M.A. Jameel, P. Peralta, C. Laird, Masing behavior in copper single crystals fatigued under load control, Materials Science and Engineering: A. 297 (2001) 48-53. doi:10.1016/S09215093(00)01275-2.

[31] Z.S. Basinski, S.J. Basinski, Fundamental aspects of low amplitude cyclic deformation in facecentred cubic crystals, Progress in Materials Science. 36 (1992) 89-148. doi:10.1016/00796425(92)90006-S.

[32] P. Lukás, L. Kunz, Role of persistent slip bands in fatigue, Philosophical Magazine. 84 (2004) $317-$ 330. doi:10.1080/14786430310001610339.

[33] P. Neumann, Coarse slip model of fatigue, Acta Metallurgica. 17 (1969) 1219-1225. doi:10.1016/0001-6160(69)90099-6.

[34] R. Neumann, P. Neumann, STRAIN BURSTS AND COARSE SLIP DURING CYCLIC DEFORMATION OF F.C.C. AND H.C.P. SINGLE CRYSTALS, Scripta Metallurgica. 4 (1970) 5.

[35] F. Lorenzo, C. Laird, Strain bursts in pure copper subjected to various forms of static and cyclic loading at ambient temperature, Materials Science and Engineering. 52 (1982) 187-194. doi:10.1016/0025-5416(82)90048-9.

[36] B.-D. Yan, C. Laird, The phenomenon of strain avalanches in cyclic deformation, Acta Metallurgica. 33 (1985) 2023-2031. doi:10.1016/0001-6160(85)90125-7.

[37] S.I. Hong, C. Laird, Cyclic Deformation Behavior of Cu- 16at.\%Al Single Crystals I: Strain Burst Behavior, Materials Science and Engineering A. 124 (1990) 183-201.

[38] N. Kiesewetter, P. Schiller, The acoustic emission from moving dislocations in aluminium, Physica Status Solidi (a). 38 (1976) 569-576. doi:10.1002/pssa.2210380218.

[39] C. Scruby, H. Wadley, J.E. Sinclair, The origin of acoustic emission during deformation of aluminium and an aluminium-magnesium alloy, Philosophical Magazine A. 44 (1981) 249-274. doi:10.1080/01418618108239532.

[40] A. Vinogradov, M. Nadtochiy, S. Hashimoto, S. Miura, Acoustic Emission Spectrum and Its Orientation Dependence in Copper Single Crystals, Materials Transactions, JIM. 36 (1995) 496503. doi:10.2320/matertrans 1989.36.496.

[41] G. L'hôte, C. Lafond, P. Steyer, S. Deschanel, T. Douillard, C. Langlois, S. Cazottes, RotationalElectron Channeling Contrast Imaging analysis of dislocation structure in fatigued copper single crystal, Scripta Materialia. 162 (2019) 103-107. doi:10.1016/j.scriptamat.2018.10.050.

[42] P. Capper, Bulk Crystal Growth: Methods and Materials, in: S. Kasap, P. Capper (Eds.), Springer Handbook of Electronic and Photonic Materials, Springer International Publishing, Cham, 2017: pp. 1-1. doi:10.1007/978-3-319-48933-9_12.

[43] D.G. Coates, Kikuchi-like reflection patterns obtained with the scanning electron microscope, The Philosophical Magazine: A Journal of Theoretical Experimental and Applied Physics. 16 (1967) 1179-1184. doi:10.1080/14786436708229968.

[44] S. Zaefferer, N.-N. Elhami, Theory and application of electron channelling contrast imaging under controlled diffraction conditions, Acta Materialia. 75 (2014) 20-50. doi:10.1016/j.actamat.2014.04.018.

[45] S. Deschanel, W. Ben Rhouma, J. Weiss, Acoustic emission multiplets as early warnings of fatigue failure in metallic materials, Scientific Reports. 7 (2017). doi:10.1038/s41598-017-13226-1.

[46] K. Mathis, F. Chmelik, Exploring Plastic Deformation of Metallic Materials by the Acoustic Emission Technique, in: W. Sikorski (Ed.), Acoustic Emission, InTech, 2012. doi:10.5772/31660.

[47] H. Liang, C.H. Woo, H. Huang, A.H.W. Ngan, T.X. Yu, Crystalline Plasticity on Copper (001), (110), and (111) Surfaces during Nanoindentation, (2004) 10.

[48] D.E.J. Armstrong, A.J. Wilkinson, S.G. Roberts, Measuring anisotropy in Young's modulus of copper using microcantilever testing, J. Mater. Res. 24 (2009) 3268-3276. doi:10.1557/jmr.2009.0396. 
[49] T. Niiyama, T. Shimokawa, Atomistic mechanisms of intermittent plasticity in metals: Dislocation avalanches and defect cluster pinning, Phys. Rev. E. 91 (2015) 022401. doi:10.1103/PhysRevE.91.022401.

[50] P. Zhang, O.U. Salman, J.-Y. Zhang, G. Liu, J. Weiss, L. Truskinovsky, J. Sun, Taming intermittent plasticity at small scales, Acta Materialia. 128 (2017) 351-364. doi:10.1016/j.actamat.2017.02.039.

[51] A. Clauset, C.R. Shalizi, M.E.J. Newman, Power-law distributions in empirical data, SIAM Review. 51 (2009) 661-703. doi:10.1137/070710111.

[52] T. Richeton, J. Weiss, F. Louchet, Dislocation avalanches: Role of temperature, grain size and strain hardening, Acta Materialia. 53 (2005) 4463-4471. doi:10.1016/j.actamat.2005.06.007.

[53] L.M. Brown, Constant intermittent flow of dislocations: central problems in plasticity, Materials Science and Technology. 28 (2014) 1209-1232. doi:10.1179/174328412x13409726212768.

[54] L.M. Brown, Power laws in dislocation plasticity, Philosophical Magazine. 96 (2016) 2696-2713. doi:10.1080/14786435.2016.1211330.

[55] R.K. Ham, The determination of dislocation densities in thin films, Philosophical Magazine. 6 (1961) 1183-1184. doi:10.1080/14786436108239679.

[56] P. Li, S.X. Li, Z.G. Wang, Z.F. Zhang, Fundamental factors on formation mechanism of dislocation arrangements in cyclically deformed fcc single crystals, Progress in Materials Science. 56 (2011) 328-377. doi:10.1016/j.pmatsci.2010.12.001.

[57] W.-W. Guo, X.-M. Wang, X.-W. Li, SEM Electron Channeling Contrast Imaging of Dislocation Structures in Fatigued [017] Cu Single Crystals Oriented for Critical Double Slip, Mater. Trans. 51 (2010) 887-891. doi:10.2320/matertrans.M2009439.

[58] D. Rouby, P. Fleischmann, Possibilités d'analyse quantitative de mécanismes irreversibles par émission acoustique, Matériaux \& Techniques. 73 (1985) 58.

[59] A. Slimani, P. Fleischmann, R. Fougères, Etude par émission acoustique de la dynamique des dislocations pendant la déformation cyclique de polycristaux d'aluminium, Journal de Physique III. 2 (1992) 933-945. doi:10.1051/jp3:1992171.

[60] F. Lorenzo, C. Laird, Strain bursts in the cyclic creep of copper single crystals at ambient temperature, Acta Metallurgica. 32 (1984) 671-680. doi:10.1016/0001-6160(84)90141-X. 